\title{
Blade Runner
}

\section{Erhard Taverna}

Dr. med., Mitglied der Redaktion

Philip K. Dick (1928-1982), ein Science-Fiction-Genie und brillanter Beobachter der amerikanischen Alltagskultur, schrieb einen Roman [1], der - 1982 von Ridley Scott mit dem Titel Blade Runner verfilmt - Kultstatus erlangte. Der Film wurde im Rahmen von Scientifica, den Wissenschaftstagen der ETH und Universität Zürich, im August im Kino Kosmos gezeigt. Die Robotik in vielen Facetten war ein Schwerpunkt zahlreicher Demonstrationen, Workshops und Vorlesungen.

Zur gleichen Zeit traten behinderte Menschen in der Bahnhofshalle Zürich gegeneinander an. Männer und Frauen, die dank Assistenzsystemen Wettkämpfe austragen. Die Piloten und Pilotinnen, so werden die Wettkämpfenden genannt, bewältigten Geschicklichkeitsparcours mit Armprothesen, Hindernisse mit Beinprothesen, motorisierten Rollstühlen und robotischen Exoskeletten. Sie lösten Alltagsprobleme wie Stufensteigen oder Wäscheklammern befestigen, mussten Gegenstände in einer Kiste erfühlen oder eine Türklinke öffnen. Ihre Leistungen wurden auf Grossleinwand übertragen und von Fachleuten und Athleten kommentiert. Eine Vorschau auf Cybathlon im Mai 2020 in der Swiss Arena in Kloten, ein Rennen in sechs Disziplinen, zusätzlich mit Gedankensteuerung im virtuellen Raum und Fahrradrennen mit Muskelstimulation, begleitet von einem wissenschaftlichen Symposium.

Die ETH Zürich gehört auf dem Feld der Robotik mit anderen zur Weltklasse. Facebook implantiert Elektroden im Schädel und misst über die Hirnaktivität die Antwort auf eine vorgegebene Auswahl an Wörtern. Samsung entwickelt eine Elektrodenkappe, die eingeschränkten Menschen ermöglichen soll, einen Fernseher zu bedienen oder ein Tablet anzusteuern. Neuralink, die Firma des Tesla- und Space-X-Gründers Elon Musk, forscht an einem Chip, der über Elektroden im Gehirn die Daten neuronaler Aktivität über einen Sender hinter dem Ohr an ein Smartphone sendet. Auf umgekehrtem Weg soll in Zukunft das Lernen revolutioniert werden. Studien zeigen, dass über ma- gnetische oder elektrische Stimulation der Musikgeschmack manipuliert oder Probanden zu riskanteren Entscheidungen gebracht werden können.

Kann man in Zukunft Menschen und Maschinen auseinanderhalten? Der Neuropsychologe Lutz Jäncke, Professor an der Universität Zürich, arbeitet im Bereich der funktionellen Neuroanatomie mit den Schwerpunkten Lernverhalten, Musikverarbeitung und Entscheidungsfindung. Er glaubt, dass diese Unterscheidung nicht mehr möglich sein wird. Der Mensch überschätze seine Einzigartigkeit masslos, eines Tages könnten ihm die Anwendungen von künstlicher Intelligenz überlegen sein.

Im Roman von Philip K. Dick jagt die Hauptfigur Rick Deckard entflohene Androiden. Seine Aufgabe ist es, als speziell ausgebildeter Polizist, die Menschheit vor der Unterwanderung durch künstliche Wesen zu bewahren. Bisher zuverlässige Testverfahren sind nicht mehr zuverlässig, da mächtige Konzerne immer perfektere Kopien herstellen, die auf extraterrestrischen Kolonien ein befristetes Dasein führen. Zudem nimmt Deckards Gefühlschaos zu, da die Verfolgten oft die besseren Menschen zu sein scheinen. Ist der erfolgreiche Prämienjäger am Ende selber ein Replikant? Medizinische Anwendungen sind das «Trojanische Pferd», das neue Technologien gesellschaftsfähig macht. Wer will sich schon gegen Stammzellen, Gentechnik und Robotik als Gegner exponieren, wenn Cochlea-Implantate und zahlreiche weitere Erfindungen ihren Nutzen bewiesen haben? Philip K. Dick führte ein chaotisches Privatleben, er war paranoid und experimentierte mit Drogen. Doch er schrieb Klassiker, die nach seinem Tod alle verfilmt wurden, darunter Total Recall, Minority Report und Blade Runner, der in der Originalausgabe anders betitelt war: Do Androids Dream of Electric Sheep?

\footnotetext{
Literatur

1 Philip K. Dick. Blade Runner, Roman, 4. Auflage. München: Heyne Verlag; 2005
} 